\title{
The Van Riper Program As Intensive Interval Therapy
}

Paper Presented at the International Fluency Association First World Congress on Fluency Disorders, Munich, Germany, August 8-12, 1994

\section{The Van Riper Program As Intensive Interval Therapy}

by Andreas Starke, M.A.

Hoeperfeld 23, 21033 Hamburg, Germany

This is a report on a therapy program for adults who stutter which has been conducted in Germany since 1987.

I have named this therapy program 'The Van Riper Program As Intensive Interval Therapy' (Van-Riper-Program als intensive Intervalltherapie). The reason for this name is that our clinical procedures for the most part follow Van Riper's recommendations as described in his book 'The Treatment of Stuttering' (1973). The word 'interval' means that we are using a block schedule. Our blocks, or segments, have a duration of five days, Monday through Friday, with six to eight weeks between the segments. One weekend, half a year later, concludes the program. We call this therapy intensive, because we work intensively (about eight hours a day) with our patients during each therapy segment.

I would like to report why we have chosen this schedule, how therapy is done, and what our results have been.

\section{Basic Considerations}

The design of this therapy program is based on the following convictions and considerations:

- We prefer to use a stuttering modification approach over a fluency shaping approach.

- We consider motivation to be a major problem in adult stuttering therapy.

- We believe that adult stuttering therapy does not require extensive involvement of a therapist.

- We consider relapse to be a major problem in adult stuttering therapy.

1. The benefits and shortcomings of both approaches, stuttering modification and fluency shaping, have been widely discussed. In my opinion, the major shortcomings are that many fluency shapers have to constantly monitor their speech to maintain fluency, many of them sound unnatural, fluency training is boring both for the patient and the therapist, and, most of all, many fluency shapers become as helpless as they had been prior to therapy in instances of great communicative or other stress. We believe that Van Riper's recommendation to teach the stutterer how to better react to his stuttering rather than to teach him to speak fluently still has its merit.

2. The lack of motivation on the part of the stuttering patient in many cases is a reflection of discouragement and skepticism. It seems wise to make therapy an interesting experience which poses new challenges throughout its course and which produces rewarding results. We try to do everything we can in order to make sure that every therapy week provides the patient with new key experiences and the feeling that he is making progress resulting from his own effort. 
3. It is our experience that the rehabilitation of a person who stutters takes two or three years when rehabilitation means that the person starts to live the life of a normal speaking person. This observation may lead to the opinion that the guidance of a therapist is needed throughout this whole process. We feel that most of what the therapist can do is to give reliable information that the patient can use for solving his problem and to arrange for certain key experiences that will help the patient to develop the skills he needs for working on his own case. This can be done in a relatively short time.

4. Compared to creating fluency by stuttering modification, it seems easy to establish speech fluency by teaching certain fluency skills (like maintaining proper subglottal air pressure, using gentle voice onsets, using light contacts in the production of consonants, and other skills), or by training a new way of speaking (reducing speed, changing the quality of voicing, overarticulating, and other strategies). But it also appears that the resulting fluency is still vulnerable to stress, and that automatization occurs neither easily nor regularly.

I want to distinguish here between failure in maintenance and relapse. Most patients who fail to maintain their fluency just give up their devices because they are tired of waiting for the automatization of fluent speech that may never occur, and because they are taught to evaluate every instance of stuttering as failure, and they are just tired of failing.

On the other hand, relapse, that is the re-occurrence of stuttering or, as we see it, the increase in the number of stuttering events and the increase in the severity of single stuttering events, seems to be a natural experience in the life of every adult person who stutters.

We feel that we have to prepare our patients so that they can learn to cope with relapses. In order to do so the patient must be given the opportunity to experience failure in his every-day life and to gain expertise in how to react to failure. A therapist who works with the patient on a regular schedule (e.g. one to three sessions per week) can more easily monitor successes and failures of a patient, and this may be one advantage of such a schedule. But if we want to make use of the advantages of working intensively with our patients and of making this therapy program a unique event in the life of the patient, as any residential program tends to be, then we have to send our patients home to their regular environment even with the limited knowledge and skills they will have acquired up to that particular point in time. At home they will struggle and fail (failure may include not struggling at all). These experiences will clarify the need for coping skills on the part of the patient and help the therapist to understand what the specific problems in applying newly learned behavior are.

\section{Procedures}

The program is carried out as a group therapy program with a group of twelve patients and two therapists in a boarding house operated by a church. My co-therapists have been Angelika Engert, Munich (1987-1990) and Bernd Koppenhagen, Wuerzburg (1991-now).

First week (Identification) The first week of therapy has two major goals, to improve the understanding of each individual patient's stuttering and to have patients understand the basic concepts of speech production.

A video recording of each patient is made on the first day of therapy in order to avoid adaptation of the patients to the setting. About ten minutes of conversational speech and five minutes of reading are recorded.

A lecture about speech production is given to the group. The purpose of this lecture is to have patients acquire a basic understanding of the following topics movement, automaticity vs. volition, quiet respiration and speech respiration, voice production, articulation of speech sounds, and coarticulation. An attempt is made to transmit the following ideas: (1) speech quality benefits more from precision than it does from the use of force, (2) stuttering is a coordination problem, (3) coordination problems are best approached by reducing speed, (4) automatic movements can be shaped by practicing, and (5) it is possible to substitute highly automatized responses by new responses that are learned as voluntary movements and 
gradually become automatized. The lecture also provides the patients with a language they can use in discussing their video recordings. This lecture is given to one half of the group while the other is being video recorded.

The video recordings are then analyzed in the presence of the entire group. In the analysis we try to distinguish between the core event of stuttering and the reaction of the stutterer to the core event. We admit that we don't know exactly what the core event is, but that it seems to be a breakdown in the servo-system of speech, a point where at least two muscles are not cooperating properly. We hypothesize that the effect of this core event could be small and that how the speaker reacts to it makes all the difference.

In some group meetings in the first week of therapy we also share the private stuttering theories of the patients and experiences the patients have had with their stuttering.

Second week (Desensitization) The major goal of the second week is to desensitize the patients to the expectation and experience of stuttering. Subgoals and procedures may vary from patient to patient. They include exercises m maintaining eye contact, analyzing and varying reactions to stuttering, observing listener behavior, manipulation of the 'point of loss of control', regaining control in a stuttering event, and exercises in gradual relaxation both in the speech mechanism and other parts of the body. For all activities pseudostuttering (both similar to and different from the habitual pattern of stuttering) is used as well as unintentional stuttering.

In this week no attempt is made to alter any aspect of speech behavior in order to improve fluency. Many patients start to make modifications in their reactions to stuttering by giving up certain devices like avoiding, postponing, interjections, starters, revisions, stop-and-repeat procedures, and others. Modification goes in the direction of 'direct stuttering' (some patients have called it 'trick-free stuttering' or 'net stuttering'). When this occurs stuttering appears to be more severe, because stuttering events are more frequent and more easily identified.

In some group meetings in the second week we share experiences that show how stuttering impairs communication. What a stutterer should do and not do in order to improve the probability of communicative success even when he stutters is discussed in all detail.

On Wednesday and Thursday one half of the group spends the day doing real-life exercises in the city and the other half is doing exercises on the telephone. Specific goals may vary from patient to patient. A common goal for all is to stay as calm as possible during a stuttering event, to stutter as directly as possible and to use certain strategies to enhance the communicative act.

We have stopped using flooding (i.e. to expose a patient to many strong stimuli until he calms down) because we have found that the experience doesn't teach the patient very much. We have replaced the slogan $>>$ Augen zu und durch! $<<$ (Close your eyes and get it behind you) by $>>$ Auger auf und langsam durch $!<<$ (Keep your eyes open and slowly work yourself though it).

Third week (Cancellation): The major goal of the third week is introduction, practice, and use of the cancellation procedure as devised by Van Riper. We start with teaching slow motion speech. Much attention is given to a proportionally precise prolongation of the natural speed production and the feel of precision as opposed to the auditory control of correctness. Next comes the use of pauses ( 3 to 5 seconds) immediately after the stuttered word, then the use of 'the pause' for preparing the fluent slow-motion production of the word, and finally the entire procedure of stuttering, pausing, preparing and slowly but strongly producing the word. More details can be found in Van Riper's text.

We encourage our patients to immediately use whatever has been taught in their spontaneous interactions with us and between themselves. We seem to be successful with this. Many patients make phone calls home during breaks and in the evening in order to test their abilities without us encouraging them to do so. 
Again, Wednesday and Thursday are used for an excursion to the city and for telephone exercises at the boarding house by one half of the group each day.

In all of our groups about one half of the patients experience substantial reduction in the frequency of stuttering in the third week, i.e. sequences of normal speech between stuttering events become longer.

Fourth week (Pull-out) The major goal of the fourth week is introduction, practice, and use of pull-outs as devised by Van Riper. The procedure is much simpler than cancellation because the patient 'only' has to gain control of the stuttering event and then complete the word making a strong slow movement through the sequence which constitutes the utterance of the rest of the word.

Most of our patients learn to use pull-outs without difficulty. Some patients need training in quickly gaining control of their stuttering events. In general patients find that using pull-outs is a pleasurable experience, many of them tend to flood their speech with pseudo-pull-outs, i.e. pull-out-like speech movements not triggered by stuttering.

We found that the need for outside activities and telephone sessions has decreased by the fourth week because many patients have enough experience in transferring new behavior to their every-day life. In order to provide an opportunity for a difficult and valuable experience patients have to give a 15-20 minute speech about a chosen topic in the fourth week as an exercise in public speaking.

By the end of the fourth week all patients with few exceptions (about one or two in a group of twelve on the average) become very fluent and produce many sentences in a row without any stuttering. For many of them (including those who have been in fluency shaping programs) this is the first time that they experience how a normal speaker feels all the time. Words flow while you think and you don't have to attend to anything concerning speech production.

Final weekend (Follow-up): It is difficult to say whether our final weekend is what Van Riper had in mind when writing about stabilization.

With our first group in 1987 we had planned for five weeks of therapy and many subsequent weekends, but, inexperienced as we were at that time, we found that there was not much more to do after the fourth week of therapy, so we dropped the fifth week. And also the following weekends appeared not to be very effective, with the exception of perhaps the first.

Now this weekend serves four purposes: (1) We review the sequence of therapy and instruct the patients to work their way backwards (pull-out, cancellation, desensitization, identification) through that sequence, as far as they have to, when they feel dissatisfied with the quality of their speech. (2) We explain the two major sources of relapse: insufficient desensitization and incomplete or deteriorating motor skills, i.e. the ability to produce quality pull-outs. We recommend considering the relative role of these two in any given situation, and teach how to determine it by testing. (3) We discuss the particular characteristics of the 'life of a stutterer' and strongly recommend to fight all tendencies to again adopt the 'stuttering life-style'. And (4) this weekend, half a year after the four-week program, certainly helps to ease the grieving process that results from dissolving the group.

\section{Results}

In 1992 we investigated the results of this program conducting a survey using a customer satisfaction study format (Starke 1993). We were able to get replies from all 60 (1987-1991) patients with the exception of 9 patients who had moved with address unknown.

Most important for us was the fact that 86 percent of all patients reported improvements in their speech, 55 percent of these consider their speech 'much improved' (sehr verbessert), and 45 percent 'somewhat improved' (etwas verbessert). All patients but one assign their improvement to this therapy program. Improvements in their private and work life were also 
remarkable, but were reported in smaller numbers. This shows that, contrary to a wide-spread opinion, a therapy program following Van Riper's guidelines is able to produce improvements not only in life quality, but also - and more clearly reported - improvements in the quality of speech. With the limited data we have we cannot conclude that the effects of therapy as reported by our patients deteriorate over time.

All our patients think that this program is worth recommending, 65 percent of our patients actually have recommended the program to others. 75 percent of our patients did not undergo any type of further therapy because of their stuttering and, based on their judgment at the time of the survey, will not do so in the future.

We feel that this therapy program meets the requirements of our patients and our own objectives to a very high degree. It is our goal to provide for our patients a set of highly effective tools for manipulating their stuttering, establishing speech fluency and coping with the inevitable relapses they have had and most likely will have in the future.

We have fewer motivational problems than usually are reported by other therapists. We feel that this is due to the combination of a programmed approach and heavy reliance on the selfresponsibility of our patients. Since our therapy is program-driven as opposed to patient-driven, our patients know that they have to comply if they don't want to take the risk of falling behind. Our drop-out rate is extremely low. In our view today further improvements will probably only concern small changes in the schedule of a particular therapy week. Most improvements will result from our ever better understanding of the stuttering problem as our patients teach us. We are learning something new from each group.

\section{References}

Starke, A. (1993). Umfrage unter Absolventen eines Therapieprogramms fuer stotternde Erwachsene. In: H. S. Johannsen, L. Springer (Eds.). Stottern: Muenster 19.-22. 5. 1993. Ulm: Verlag Phoniatrische Ambulanz.

Van Riper, C. (1973). The Treatment Of Stuttering. Englewood Cliffs, N.J.: Prentice Hall Van Riper, C. (1986). Die Behandlung des Stotterns Koeln Bundesvereinigung StottererSelbsthilfe e.V.

added July 1,1998 on

http://www.mankato.msus.edu/dept/comdis/kuster/conference/starke1.html

Andreas Starke, Speech-Language Pathologist

Hoeperfeld 23

21033 Hamburg

Germany

Phone +49-(0)40-724 10007

Fax +49-(0)40-724 10008

Email: info@andreasstarke.de 